EPJ Web of Conferences 29, 00041 (2012)

DOI: 10.1051/epjconf/20122900041

CC Owned by the authors, published by EDP Sciences, 2012

\title{
Thermal resistance investigation of the giant magneto- resistance thin layers by the PTD technique
}

\author{
T. Ghrib ${ }^{1, a}{ }^{*}$, M.S. Ben Kraîem ${ }^{2}$, C. Bordel $^{3}$, N.Yacoubi ${ }^{1}$ and A. Cheikhrouhou ${ }^{2}$ \\ ${ }^{1}$ Photothermal Laboratory, IPEIN, 8000 Nabeul, TUNISIA. \\ ${ }^{2}$ Laboratoire de Physique des Matériaux, Faculté des Sciences de Sfax, B.P. 1171 - 3000 Sfax, \\ TUNISIE. \\ ${ }^{3}$ Groupe de Physique des Matériaux UMR CNRS 6634, BP.12, Saint Etienne du Rouvray Cedex, \\ FRANCE.
}

\begin{abstract}
In this paper, we present a thermal properties investigation of giant magnetoresistance $\mathrm{Mn} / \mathrm{Fe}$ layers as a function of Mn thickness using the Photothermal Deflexion Technique (PTD). We observe that the thermal resistance reaches its maximum for a $\mathrm{Mn}$ critical thickness corresponding to the antiparallel ferromagnetic coupling.
\end{abstract}

Keywords: giant magneto-resistance, thermal resistance, photothermal deflexion technique.

\section{Introduction}

Since the 1980's many researches have been carried out on the magnetic multi-layers containing ferromagnetic and nonmagnetic materials. In October 2007, the Nobel Prize in Physics was discerned to Albert Fert and Peter Grünberg for the Giant magneto-resistance (GMR) effect observed in magnetic multi-layers [1-3].

The GMR samples are an assembly of multi-layers alternated by ferromagnetic-metal and nonmagnetic-metal (FM/NM) with a thickness of about $1 \mathrm{~nm}$.

One of the first manifestations of the new properties of these structures was the observation, in 1986, of an antiferromagnetic coupling between layers of iron in three layers $\mathrm{Fe} / \mathrm{Cr} / \mathrm{Fe}$ epitaxially deposited by molecular jets on AsGa substrate [2].

Several theorists such as Camley, Trigui, Barthélémy [4-7] tried to develop mathematical models allowing the interpretation of these multi-layers. Duvail et al. [8] were interested in the dependence versus temperature and thickness of the resistivity and the magneto-resistance of $\mathrm{Co} / \mathrm{Cu}$ multi- layer. Over the past 30 years a large interest of the GMR samples has been motivated by the understanding of their very magnetic properties, which are briefly described as follow: the ferromagnetic-metal is microscopically formed by small zones called Weiss-zones. Each zone is characterized by magnetic moments aimed at in the same direction. In the case of a small lateral dimension, a magnetic interaction between the ferromagnetic layers giving a global magnetization oriented in the same direction, one says for this balance situation that there is a parallel coupling between the magneticlayers. By increasing the thickness of the nonmagnetic-metal layer, one crosses a value which,

\footnotetext{
a e-mail : taher.ghrib@yahoo.fr
} 
beyond the coupling, becomes antiparallel. The resistance is higher for antiparallel configuration and smaller for parallel magnetization configuration.

In this work, we studied the effect of Mn layer thickness on the thermal properties of the GMR samples constituted by an assembly of alternated Manganese thin layers (nonmagnetic metal) with a thickness varying from 0.3 to $1.7 \mu \mathrm{m}$ and $1.5 \mu \mathrm{m}$ iron layer (ferromagnetic metal).

The thermal properties were determined by the Photo-Thermal Deflection (PTD) technique [8-10].

\section{Sample preparation}

The evaporation enclosure is equipped with a turbo-molecular pump, allowing a vacuum of about $10^{-}$ ${ }^{9}$ mbar. The materials to evaporate ( $\mathrm{Mn}$ and $\mathrm{Fe}$ ) are placed in alumina crucibles and are heated by radiation up to $1250^{\circ} \mathrm{C}$. Each overflowing cell is equipped with an electro-pneumatic mask, which makes it possible to deposit alternatively the various materials without stopping their evaporations.

These layers are deposited with evaporation rates of $2.10^{-12} \mathrm{~m} \cdot \mathrm{s}^{-1}$ for iron and $6.10^{-12} \mathrm{~m} \cdot \mathrm{s}^{-1}$ for manganese. The thickness of each layer is measured via a quartz oscillator calibrated at the deposition temperature. We obtain an assembling composed of 21 identical layers deposited on silicon substrate: $\mathrm{Si} / \mathrm{Mn} / \mathrm{Fe} / \mathrm{Mn} / \ldots \ldots . \mathrm{Fe} / \mathrm{Mn}$. The protective layer is Mn with $22 \mathrm{~nm}$ thickness.

\section{Magnetic properties}

From magnetization measurements recorded versus magnetic applied field, we have plotted the hysteresis loops (Fig. 1). We can noticed that, the larger value of Mn thickness is, the more the cycles are flat, i.e., more need for applying a large field $\mathrm{H}$ to reach the saturation is required. This behavior has been observed for an antiparallel coupling. Whereas for thin Mn layers we observe square cycles, signature of parallel manganese ions coupling.

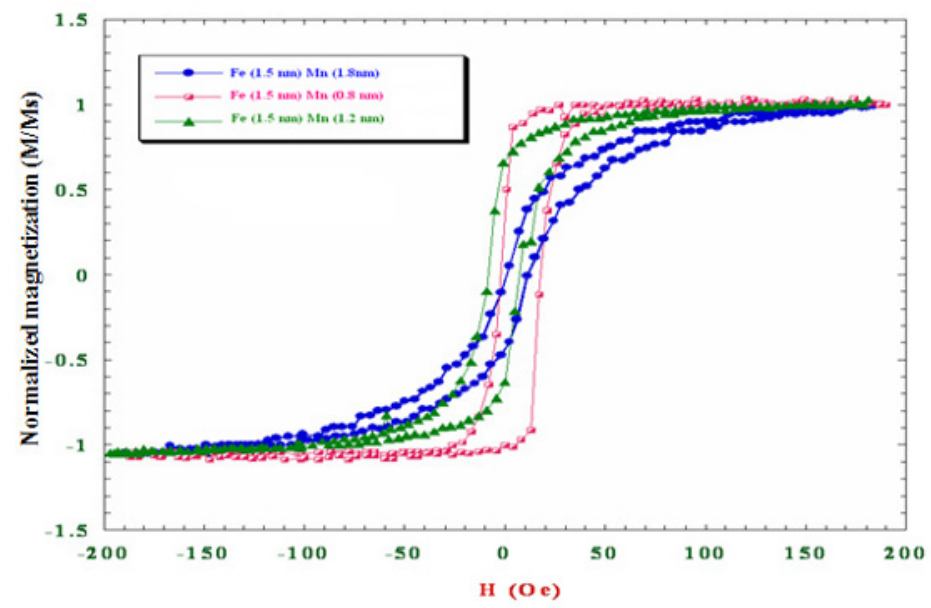

Fig.1. $\mathrm{M}(\mathrm{H})$ loops for Fe/Mn GMR samples corresponding to 0.8, 1.2 and 1.8nm thickness of the Mn layer

\section{Thermal properties}

The thermal properties such as the thermal conductivity and the thermal properties are determined by the PTD technique. 


\subsection{Principle of the PTD technique}

This method consists in heating a sample with a modulated light beam of intensity $\mathrm{I}=\mathrm{I}_{0}(1+\cos \omega t)$. The thermal wave generated by the optical absorption of the sample will propagate in the sample and in the surrounding fluid (air in our case). The thermal wave in the fluid will induce a temperature gradient then a refractive index gradient in the fluid which will cause the deflection $\psi$ of a probe Laser beam skimming the sample surface. This deflection may be related to the thermal properties of the sample.

The sample is a stack of 21 layers (Fig. 2), We write the heat equations in these areas and in the two surrounding fluid by designating $\mathrm{K}_{\mathrm{i}}, \mathrm{D}_{\mathrm{i}}$ and $\mathrm{l}_{\mathrm{i}}$ respectively the thermal conductivity, the thermal Diffusivity and the thickness of the layer $\mathrm{i}$.

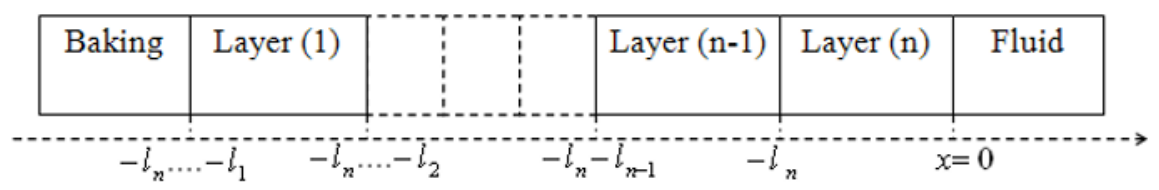

Fig.2. Different medias browsed by the heat

\subsection{Theoretical model}

\subsubsection{Probe beam deflection}

In the case of a uniform heating we can use a 1-dimensional approximation, and the amplitude $|\psi|$ and phase $\varphi$ of the probe beam deflection $\psi$ are given by:

$$
|\psi|=\frac{\sqrt{2} L}{n \mu_{f}} \frac{d n}{d T_{f}}\left|T_{0}\right| e^{-\frac{x}{\mu_{f}}} \text { and } \varphi=-\frac{x}{\mu_{f}}+\theta+\frac{5 \pi}{4}
$$

Where 1 is the width of the pump beam in the direction of the probe laser beam, $\mathrm{n}, \mu_{f}$ and $\mathrm{T}_{\mathrm{f}}$ are respectively the refractive index, the thermal diffusion length and the temperature of the fluid. $\left|T_{0}\right|$ and $\theta$ are respectively the amplitude and phase of the temperature $\mathrm{T}_{0}$ at the sample surface which are function of the thermal properties of the different media. $\mathrm{x}$ is the distance between the probe beam axe and the sample surface.

Before the calculation of the probe beam deflection, one must know the expression of the surface temperature $\mathrm{T}_{0}$ that is calculated as follows.

\subsubsection{Surface temperature}

The resolution of the heat equation gives the following temperature equation: 
EPJ Web of Conferences

$$
\begin{aligned}
& T_{f}(x, t)=T_{0} e^{-\sigma_{f} x} e^{j \omega t} \\
& T_{n}(x, t)=\left(X_{n} e^{\sigma_{n} x}+Y_{n} e^{-\sigma_{n} x}-E_{n} e^{\alpha_{n} x}\right) e^{j \omega t} \\
& T_{n-1}(x, t)=\left(X_{n-1} e^{\sigma_{n-1}\left(x+l_{n}\right)}+Y_{n-1} e^{-\sigma_{n-1}\left(x+l_{n}\right)}-E_{n-1} e^{\alpha_{n-1}\left(x+l_{n}\right)}\right) e^{j \omega t} \\
& \text { if } \quad 0 \leq x \leq l_{f} \\
& \text { if } \quad-l_{n} \leq x \leq 0 \\
& \text { if } \quad-l_{n}-l_{n-1} \leq x \leq-l_{n-1} \\
& \begin{array}{lr}
T_{1}(x, t)=\left(X_{1} e^{\sigma_{1}\left(x+l_{n}+l_{n-1} \ldots+l_{2}\right)}+Y_{1} e^{-\sigma_{1}\left(x+l_{n}+l_{n-1} \ldots+l_{2}\right)}-E_{1} e^{\alpha_{1}\left(x+l_{n}+\ldots+l_{2}\right)}\right) e^{j \omega t} & \text { if } \quad-l_{n}-l_{n-1} \ldots-l_{1} \leq x \leq-l_{n}-l_{n-1} \ldots-l_{2} \\
T_{b}(x, t)=W e^{\sigma_{b}\left(x+l_{n}+\ldots+l_{2}+l_{1}\right)} e^{j \omega t} & \text { if }-l_{n}-l_{n-1} \ldots-l_{1}-l_{b} \leq x \leq-l_{n}-l_{n-1} \ldots-l_{1}
\end{array}
\end{aligned}
$$

We can then write the flow equation in each medium

$$
\begin{array}{lrr}
\phi_{f}(x, t)=K_{f} \sigma_{f} T_{0} e^{-\sigma_{f} x} e^{j \omega t} & \text { if } & 0 \leq x \leq l_{f} \\
\phi_{n}(x, t)=-K_{n} \sigma_{n}\left(X_{n} e^{\sigma_{n} x}-Y_{n} e^{-\sigma_{n} x} \frac{\alpha_{n}}{\sigma_{n}} E_{n} e^{\alpha_{n} x}\right) e^{j \omega t} & \text { if } \quad-l_{n} \leq x \leq 0 \\
\phi_{n-1}(x, t)=-K_{n-1} \sigma_{n-1}\left(X_{n-1} e^{\sigma_{n-1}\left(x+l_{n}\right)}-Y_{n-1} e^{-\sigma_{n-1}\left(x+l_{n}\right)} \frac{\alpha_{n-1}}{\sigma_{n-1}} E_{n-1} e^{\alpha_{n-1}\left(x+l_{n}\right)}\right) e^{j \omega t} & \text { if } & -l_{n}-l_{n-1} \leq x \leq-l_{n-1} \\
& & \\
\phi_{1}(x, t)=-K_{1} \sigma_{1}\left(X_{1} e^{\sigma_{1}\left(x+l_{n}+l_{n-1} \ldots+l_{2}\right)}-Y_{1} e^{-\sigma_{1}\left(x+l_{n}+l_{n-1} \ldots+l_{2}\right)}-\frac{\alpha_{1}}{\sigma_{1}} E_{1} e^{\alpha_{1}\left(x+l_{n}+\ldots+l_{2}\right)}\right) e^{j \omega t} & \text { if } & -l_{n}-l_{n-1} \ldots-l_{1} \leq x \leq-l_{n}-l_{n-1} \ldots-l_{2} \\
\phi_{b}(x, t)=-K_{b} \sigma_{b} W e^{\sigma_{b}\left(x+l_{n}+\ldots .+l_{2}+l_{1}\right)} e^{j \omega t} & \text { if }-l_{n}-l_{n-1} \ldots-l_{1}-l_{b} \leq x \leq-l_{n}-l_{n-1} \ldots-l_{1}
\end{array}
$$

The temperature and heat flow continuity at the interface $\mathrm{x}=$-ln permit to obtain:

$$
\begin{aligned}
& \left(\begin{array}{ccc}
1 & 1 & -1 \\
1 & -1 & -r_{n-1} \\
0 & 0 & E_{n} / E_{n-1}
\end{array}\right)\left(\begin{array}{l}
X_{n-1} \\
Y_{n-1} \\
E_{n-1}
\end{array}\right)=\left(\begin{array}{ccc}
e^{-\sigma_{n} l_{n}} & e^{\sigma_{n} l_{n}} & -e^{-\alpha_{n} l_{n}} \\
c_{n} e^{-\sigma_{n} l_{n}} & -c_{n} e^{\sigma_{n} l_{n}} & -c_{n} r_{n} e^{-\alpha_{n} l_{n}} \\
0 & 0 & 1
\end{array}\right)\left(\begin{array}{l}
X_{n} \\
Y_{n} \\
E_{n}
\end{array}\right) \\
& G_{n}\left(\begin{array}{c}
X_{n-1} \\
Y_{n-1} \\
E_{n-1}
\end{array}\right)=D_{n}\left(\begin{array}{c}
X_{n} \\
Y_{n} \\
E_{n}
\end{array}\right) \text { or }\left(\begin{array}{c}
X_{n-1} \\
Y_{n-1} \\
E_{n-1}
\end{array}\right)=G_{n}^{1} \cdot D_{n}\left(\begin{array}{l}
X_{n} \\
Y_{n} \\
E_{n}
\end{array}\right)=M_{n}\left(\begin{array}{c}
X_{n} \\
Y_{n} \\
E_{n}
\end{array}\right) \\
& \text { Where } G_{n}=\left(\begin{array}{ccc}
1 & 1 & -1 \\
1 & -1 & -r_{n-1} \\
0 & 0 & E_{n} / E_{n-1}
\end{array}\right) \text {, } \\
& D_{n}=\left(\begin{array}{ccc}
e^{-\sigma_{n} l_{n}} & e^{\sigma_{n} l_{n}} & -e^{-\alpha_{n} l_{n}} \\
c_{n} e^{-\sigma_{n} l_{n}} & -c_{n} e^{\sigma_{n} l_{n}} & -c_{n} r_{n} e^{-\alpha_{n} l_{n}} \\
0 & 0 & 1
\end{array}\right) \text { and } M_{n}=G_{n}^{-1} \cdot D_{n} .
\end{aligned}
$$

In the same way we can write in the interface $\mathrm{x}_{\mathrm{i}}=-\mathrm{1}_{\mathrm{n}}-1_{\mathrm{n}-1} \ldots-1_{\mathrm{i}}\left(\begin{array}{c}X_{i-1} \\ Y_{i-1} \\ E_{i-1}\end{array}\right)=M_{i}\left(\begin{array}{c}X_{i} \\ Y_{i} \\ E_{i}\end{array}\right)$ 
Then $\left(\begin{array}{l}X_{1} \\ Y_{1} \\ E_{1}\end{array}\right)=M_{2} \cdot M_{3} \ldots . M_{n}\left(\begin{array}{l}X_{n} \\ Y_{n} \\ E_{n}\end{array}\right)=\left(\begin{array}{lll}m_{11} & m_{12} & m_{13} \\ m_{21} & m_{22} & m_{23} \\ m_{31} & m_{32} & m_{33}\end{array}\right)\left(\begin{array}{c}X_{n} \\ Y_{n} \\ E_{n}\end{array}\right)$

In this case we write $\left\{\begin{array}{l}X_{1}=m_{11} X_{n}+m_{12} Y_{n}+m_{13} E_{n} \\ Y_{1}=m_{21} X_{n}+m_{22} Y_{n}+m_{23} E_{n}\end{array}\right.$

By writing of the heat flow and temperature continuity at the interfaces $x=0$ and $x=-l_{3}-l_{2}-l_{1}$, we obtain respectively:

$X_{n}=\frac{1}{2}(1-g) T_{0}+\left(1+r_{3}\right) \frac{E_{n}}{2}, Y_{n}=\frac{1}{2}(1+g) T_{0}+\left(1-r_{3}\right) \frac{E_{n}}{2}$.

And $(1-b) e^{-\sigma_{1} l_{1}} X_{1}-(1+b) e^{\sigma_{1} l_{1}} Y_{1}-\left(r_{1}-b\right) e^{-\alpha_{1} l_{1}} E_{1}=0$

Then $X_{1}=\frac{m_{11}}{2}\left((1-g) T_{0}+\left(1+r_{n}\right) E_{n}\right)+\frac{m_{12}}{2}\left((1+g) T_{0}+\left(1-r_{n}\right) E_{n}\right)+m_{13} E_{n}$ and

$$
Y_{1}=\frac{m_{21}}{2}\left((1-g) T_{0}+\left(1+r_{n}\right) E_{n}\right)+\frac{m_{22}}{2}\left((1+g) T_{0}+\left(1-r_{n}\right) E_{n}\right)+m_{23} E_{n} .
$$

Or too $X_{1}=\left(m_{11}(1-g)+m_{12}(1+g)\right) \frac{T_{0}}{2}+\left(m_{11}\left(1+r_{n}\right)+m_{12}\left(1-r_{n}\right)+2 m_{13}\right) \frac{E_{n}}{2}$ and $Y_{1}=\left(m_{21}(1-g)+m_{22}(1+g)\right) \frac{T_{0}}{2}+\left(m_{21}\left(1+r_{n}\right)+m_{22}\left(1-r_{n}\right)+2 m_{23}\right) \frac{E_{n}}{2}$.

That is $X_{1}=\eta_{1} T_{0}+\eta_{2} E_{n}$ and $Y_{1}=\eta_{3} T_{0}+\eta_{4} E_{n}$

By replacing $\mathrm{X}_{1}$ and $Y_{1}$ by their expressions in equation (1) one obtains:

$$
(1-b) e^{-\sigma_{1} l_{1}}\left(\eta_{1} T_{0}+\eta_{2} E_{n}\right)-(1+b) e^{\sigma_{1} l_{1}}\left(\eta_{3} T_{0}+\eta_{4} E_{n}\right)-\left(r_{1}-b\right) e^{-\alpha_{1} l_{1}} E_{1}=0
$$

What gives

$$
\left((1-b) \eta_{1} e^{-\sigma_{1} l_{1}}-(1+b) \eta_{3} e^{\sigma_{1} l_{1}}\right) T_{0}=\left((1+b) \eta_{4} e^{\sigma_{1} l_{1}}-(1-b) \eta_{2} e^{-\sigma_{1} l_{1}}\right) E_{n}+\left(r_{1}-b\right) e^{-\alpha_{1} l_{1}} E_{1}
$$

Finally

$$
T_{0}=\left[\left((1+b) \eta_{4} e^{\sigma_{1} l_{1}}-(1-b) \eta_{2} e^{-\sigma_{1} l_{1}}\right) E_{n}+\left(r_{1}-b\right) e^{-\alpha_{1} l_{1}} E_{1}\right] /\left[(1-b) \eta_{1} e^{-\sigma_{1} l_{1}}-(1+b) \eta_{3} e^{\sigma_{1} l_{1}}\right]
$$

With $E_{i}=\frac{\alpha_{i}}{2 K_{c i}\left(\alpha_{i}^{2}-\sigma_{i}^{2}\right)}$

\subsection{Experimental set-up}

The sample is heated by a halogen lamp light of $100 \mathrm{~W}$ power modulated thanks to a mechanical chopper at a variable frequency. A (He-Ne) Laser probe beam skimming the sample surface at a distance $\mathrm{z}$ is deflected. This deflection can be detected by a four quadrant photo-detector and converted into an electrical signal which is measured by a lock-in amplifier. Through the intermediary of interfaces, the mechanical chopper and the Look-in amplifier a microcomputer will set the desired modulation frequency and read the values of the amplitude and phase of the photothermal signal and then draw their variations according to the square root modulation frequency (Fig. $3)$. 


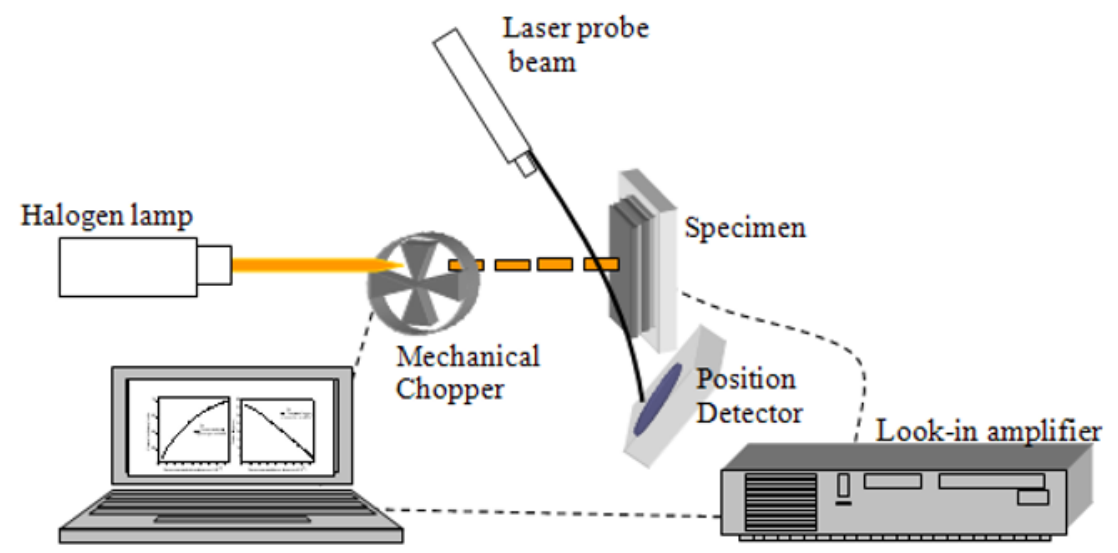

Fig. 3. Schematic experimental setup

\subsection{Determination of the thermal properties}

In order to determine the thermal properties of the seven samples, we have plotted in figure 4 the experimental variation of phase and normalized amplitude of the PTD signal versus the square root modulation frequency. The difference between these curves is attributed to the difference of their thermal conductivity, thermal diffusivity and absorption coefficient. Using the theoretical model presented in section 2 one can deduce this thermal properties $\left(\mathrm{K}_{\mathrm{i}}, \mathrm{D}_{\mathrm{i}}\right)$ and optical absorption $\alpha_{i}$.
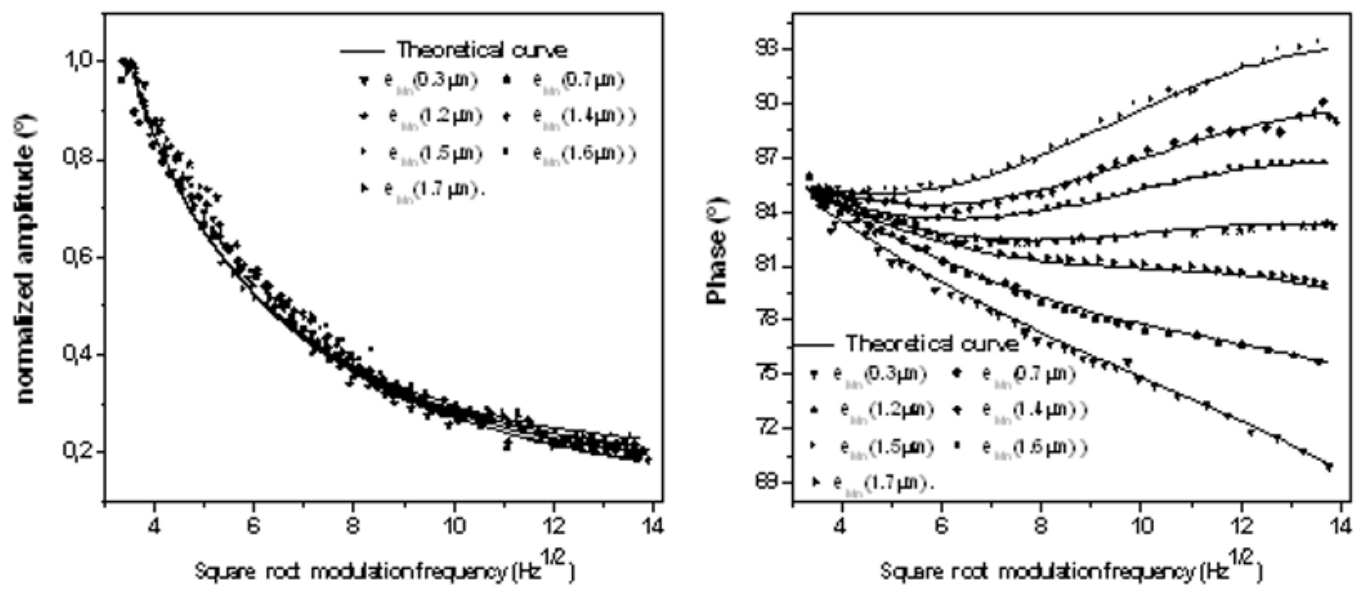

Fig.4. Normalized amplitude and phase of the photothermal signal versus the square root modulation frequency for different Mn thickness.

Table 1 illustrates the thermal conductivities, the thermal diffusivities and the absorption coefficients of the Mn and Fe layers in the GMR samples for all manganese thicknesses. We notice that only thermal conductivities of the materials and the Mn absorption coefficient vary by increasing the Mn thickness. 
Table1: Evolution of the thermal conductivities, thermal diffusivities and absorption coefficient of the Fe and Mn layer in the GMR samples for various Mn thickness.

\begin{tabular}{|l|l|l|l|l|l|l|l|}
\hline \multicolumn{2}{|l|}{$\begin{array}{l}\text { thickness } \\
(\mathrm{nm})\end{array}$} & \multicolumn{2}{l}{$\begin{array}{l}\text { Thermal conductivity } \\
(\text { W.m-1.K-1) }\end{array}$} & \multicolumn{2}{l}{$\begin{array}{l}\text { Thermal diffusivity } \\
(10-4 \mathrm{~m} 2 . \mathrm{s}-1)\end{array}$} & $\begin{array}{l}\text { Absorption coefficient } \\
(\mathrm{m}-1)\end{array}$ \\
\hline $\mathrm{Fe}$ & $\mathrm{Mn}$ & $\mathrm{Fe}$ & $\mathrm{Mn}$ & $\mathrm{Fe}$ & $\mathrm{Mn}$ & $\mathrm{Fe}$ & Mn \\
\hline 1.5 & 0.3 & 0.36 & 0.05 & 1.5 & 0.3 & 0.36 & 0.05 \\
\hline 1.5 & 0.7 & 0.34 & 0.032 & 1.5 & 0.7 & 0.34 & 0.032 \\
\hline 1.5 & 1.2 & 0.31 & 0.013 & 1.5 & 1.2 & 0.31 & 0.013 \\
\hline 1.5 & 1.4 & 0.19 & 0.0076 & 1.5 & 1.4 & 0.19 & 0.0076 \\
\hline 1.5 & 1.5 & 0.084 & 0.0064 & 1.5 & 1.5 & 0.084 & 0.0064 \\
\hline 1.5 & 1.6 & 0.32 & 0.0092 & 1.5 & 1.6 & 0.32 & 0.0092 \\
\hline 1.5 & 1.7 & 1.13 & 0.03 & 1.5 & 1.7 & 1.13 & 0.03 \\
\hline
\end{tabular}

We can see from this table that only the thermal conductivities of both layers and the absorption coefficient of Mn layers vary with varying the thickness of the Mn layer.

The thermal resistance of the sample is given by $R_{t h}=\frac{1}{S} \sum_{i} \frac{e_{i}}{K_{i}}$. Where $e_{i}, S$ and $K_{i}$ are respectively the thickness, the surface and the thermal conductivity of the sample and for a surface equal to $S=1 \mathrm{~cm}^{2}$ we can trace the evolution of the thermal resistance of the GMR materials versus the Mn thickness as shown in Figure 5. We notice from these curves that the thermal resistance and the $\mathrm{Mn}$ absorption coefficient present maximum values for a $\mathrm{Mn}$ thickness equal to $1.5 \mathrm{~nm}$. According to the theoretical study proposed in section IV, this thickness correspond to the transition ferromagnetic parallel-antiparallel and more precisely corresponds to an antiparallel coupling of the dipoles. Several experimental studies [1-3, 7, 9] showed that the magneto-resistance also reaches a maximum value for a critical thickness of nonmagnetic material. Thus one can deduce a mathematical relation which relates the magnetic and thermal resistances similar to that proposed by Wiedemann-Franz [10] $K_{e}=\sigma L T$ giving a relationship between the electronic conductivity of the heat $K_{e}$ and the electric conductivity $\sigma$, where $L=2.4 \times 10^{-8}$ USI and $\mathrm{T}$ is the sample temperature, which shows that the two parameters have the same evolution.
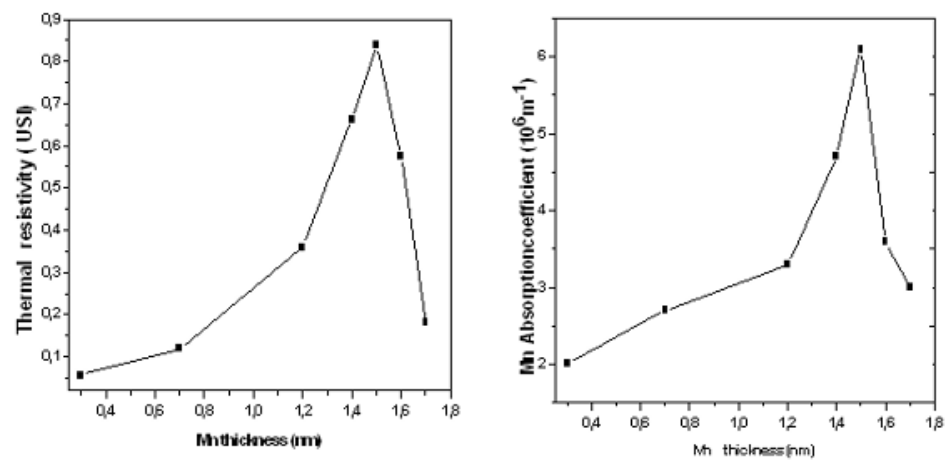

Fig.5. Thermal resistivity and Mn absorption coefficient of the GMR samples evolution with the Mn thickness 222 


\section{Conclusion}

In this work we have studied the evolution of the thermal properties of the giant magneto-resistance samples made of an assembly of alternated $\mathrm{Mn} / \mathrm{Fe}$ layers using photothermal deflection technique. A developed mathematical model has been given. We have also been interested in the thermal resistance evolution as a function of Mn thickness, and we have noticed that it reaches a maximum value for a $\mathrm{Mn}$ critical thickness corresponding to an antiparallel ferromagnetic coupling.

\section{References}

1. M. N. Baibich, J. M. Broto, A. Fert, F. Nguyen Van Dau, F. Petroff, P. Etienne, G. Creuzet, A. Friederich et J. Chazelas, Phys. Rev. Lett. 612472 (1988).

2. P. Grünberg, R. Schreiber, Y. Pang, M. B. Brodsky and H. Sowers Phys. Rev. Lett. 572442 (1986).

3. G. Binash, P. Grünberg, F. Saurenbach and W. Zinn, Phys. Rev. B 394828 (1989).

4. R. E. Camley et J. Barnas Phys. Rev. Lett. 63664 (1989).

5. F. Trigui, E. Velu, et C. Dupas, J. Magn. Magn. Mater. 93421 (1991).

6. A. Barthélémy et A. Fert, Phys. Rev. B 43, 13,124 (1991).

7. ZHAO Pingbo, LI Bozang, PU Fuke, CHIN.PHYS.LETT, Vo1.12, No.8 485 (1995).

8. J. L. Duvail, A. Fert, L. G. Pereira, et D. K. Lottis, J. Appl. Phys. 757070 (1994).

9. S. N. Ohno and K. Inomate, Phys. Rev. Lett. 721553 (1994).

10. X. J. Liu, Q. J. Huang, S. Y. Zhang, A. H. Luo, C. X. Zhao, J. Phys. Chem. Solids 65, 1247 (2004). 